\title{
Development and Verification of Simulation Model Based on Real MANET Experiments for Transport Layer Protocols (UDP and TCP)
}

\author{
Joarder Mohammad Mustafa Kamal ${ }^{1} \quad$ Mohammad Shahidul Hasan $^{1}$ \\ Alison L. Griffiths ${ }^{1} \quad$ Hongnian $\mathrm{Yu}^{2}$ \\ ${ }^{1}$ Faculty of Computing, Engineering and Technology, Staffordshire University, ST18 0AD, UK \\ ${ }^{2}$ School of Design, Engineering and Computing, Bournemouth University, BH12 5BB, UK
}

\begin{abstract}
There is a lack of appropriate guidelines for realistic user traces, mobility models, routing protocols, considerations of real-life challenges, etc. for general-purpose mobile ad hoc networks (MANET). In this paper, four laptops are used in an open field environment in four scenarios to evaluate the performances of Internet control message protocol (ICMP) based ping and transmission control protocol (TCP) based streaming video applications using optimised link state routing (OLSR) implementation in an IEEE 802.11g wireless network. Corresponding simulations are developed in Network Simulator ns-2 by setting simulation parameters according to the real experiments. Difficulties faced to regenerate real-life scenarios have been discussed and the gaps between reality and simulation are identified. A setup guideline to produce realistic simulation results has been established.
\end{abstract}

Keywords: Mobile ad-hoc networks (MANET), real-life experiment, Internet control message protocol (ICMP), user datagram protocol (UDP), transmission control protocol (TCP), optimised link state routing (OLSR), ns-2.

\section{Introduction}

Mobile ad hoc network (MANET) is one of the most theoretically researched areas of multi-hop ad hoc networking. It is composed of mobile network objects, e.g., IEEE 802.11-based wireless local area network (WLAN), ZigBee, etc. with truly dynamic and uncertain mobility where no network infrastructure pre-exists. Nowadays, MANETs are used in different specialised applications, such as control, logistics and automation, surveillance and security, transportation management, battlefields, environmental monitoring, unexplored and hazardous conditions, home networking. However, there is no unified specification or standard for the critical internet working aspects, e.g., addressing schemes, topology control, routing mechanisms, crosslayer interactions between different protocols, quality of service (QoS) support related to this technology. Researchers often examine large scale of MANET topology with fully decentralised control ${ }^{[1]}$. In reality, it is often seen that MANET nodes sometimes choose unreliable long wireless paths over short multi-hop paths and generate intolerable packet drop counts resulting performance degradation. Again, real-world experimentations vary in a significant way when compared with the simulation works. Due to the lack of appropriate guidelines for realistic user traces, mobility models, routing protocols and considering real-life challenges, it is difficult to reproduce any typical scenario in reality apart from simulation. Therefore, researchers have focused on proof-of-concept strategies and have built experimental prototypes for testing in real world ${ }^{[2]}$.

In this paper, an evaluation of real MANET experiments

Manuscript received March 15, 2011; revised May 9, 2011

This work was supported by eLINK project, EU Erasmus Mundus External Cooperation Window - Asia Reginal Call (EMECWNo. 149674-EM-1-2008-1-UK-ERAMUNDUS). with four nodes has been presented and corresponding simulations have been developed according to the real experiment values to identify the differences in the results. The initial results are reported by Kamal et al. ${ }^{[3]}$ that considers only one scenario with static nodes. On the other hand, this paper investigates four different scenarios with both static and mobile nodes. To compare the performances of real experiment and simulation, both transmission control protocol (TCP) and user datagram protocol (UDP) are investigated. A concise guideline is established to minimise the differences for future research.

The rest of the paper is organised as follows. In Section 2, a brief literature review is provided on the real experimentations conducted in various researches. Section 3 discusses the real-time MANET experimentations and the corresponding simulations utilising real-world parameters and settings. Section 4 presents the real experiment results and comparison with the corresponding simulations. Section 5 summarises the lessons learnt from the experiments and simulations. Finally, Section 6 provides the conclusions and the future work.

\section{Background and related work}

Many MANET issues such as complex network topology, asymmetric communication links, rapid link quality change, constant reliability of links are difficult to control or even cannot be controlled in real-life situations ${ }^{[2]}$. Conti et al. ${ }^{[1,4]}$ have made an extensive review on the differences between theoretical research and reality of this prominent technology. An experimental setup has been made to evaluate the performance of dynamic source routing (DSR $)^{[5]}$ prototype which consists of five mobile nodes with global 
positioning system (GPS) installed on cars moving at variable speeds ${ }^{[6]}$. Two stationary mobile IP nodes are also placed $671 \mathrm{~m}$ apart from the opposite ends of the vehicle travel path and exchange Internet control message protocol (ICMP) packets via five multi-hop mobile nodes. Another real-time MANET experiment has been conducted with four fixed and one mobile node with IEEE $802.11 \mathrm{~b}$ network adapters, fixed $1 \mathrm{Mbps}$ data rate and $5 \mathrm{~m}$ transmission range $^{[7]}$. The experiment suggests that choosing unreliable direct links rather than multi-hop reliable links results in poor performance of MANET routing protocols. Some 100 autonomous mobile robots are used to create a test-bed consisting of a maximum five-hop MANET with $1 \mathrm{Mbps}$ throughput ${ }^{[8]}$. The network breaks down when all the nodes want to join at the same time which happens because of unreliable routing protocol implementation. Three static nodes with onboard GPS and IEEE 802.11b network interface are used to evaluate the performance of greedy perimeter stateless routing (GPSR) protocol ${ }^{[9]}$ and the investigation exhibits $400 \mathrm{Kbps}$ throughput and some lost routing broadcast packets. A comparison of four MANET routing protocols has been performed with 33 mobile nodes which finds high overhead of control packets for reactive protocols than the proactive ones ${ }^{[10]}$. The original dynamic source routing (DSR) protocol implementation from the popular network simulator ns-2 ${ }^{[11]}$ is used in a real-life MANET with four static and two mobile nodes in [12]. The average packet delivery ratio is $95 \%$ with overall latency of $30 \mathrm{~ms}$ which justifies the ns-2 implementation. Another DSR implementation is tested with 10 nodes where some nodes are remote-controlled mini planes ${ }^{[13]}$. The experiment has achieved $250 \mathrm{Kbps}$ throughput with $30 \mathrm{~ms}$ latency over a maximum three hop MANET. Twenty cars equipped with four directional antennas and IEEE 802.11b network interface are used in an evaluation of a link state routing protocol hazy-sighted link state (HSLS) ${ }^{[14]}$ at Bolt, Beranek and Newman (BBN) Technologies, Cambridge ${ }^{[15]}$. The experiment has outperformed similar type of experiment using Optimised Link State Routing (OLSR) protocol ${ }^{[16]}$. In another experiment, eight nodes are used to examine ad hoc on-demand distance vector (AODV) routing ${ }^{[17]}$ and OLSR routing protocols with a peer-to-peer $(\mathrm{P} 2 \mathrm{P})$ networking system CrossROAD ${ }^{[18]}$. In the experiments, it is found that the CrossROAD over OLSR outperforms traditional P2P systems over AODV. Some 9 to 37 nodes are used within four mobility groups indoor on AODV and OLSR protocols, and it is found that the approach of choreographing node movement is suitable for real-life MANET testing ${ }^{[19]}$. An investigation has been carried out on the throughput of the IEEE 802.11 distributed coordination function considering a number of factors, e.g., path loss, multi-path fading ${ }^{[20]}$. $\mathrm{Wu}$ et al. ${ }^{[21]}$ analysed the reliability of multihop wireless networks which can suffer from node failure and random channel fading ${ }^{[22]}$. A discrete system model based on IEEE 802.11b has been developed in [22] for packet transmission process, packet dropout sequence and network scheduling. Although years of experiments have been carried out, a well-justified methodology, network architecture and benchmarks are yet to be defined.

\section{Real-life MANET experiments and corresponding simulations}

\subsection{Real-life MANET scenarios and cases}

Commonly used node movements are "end and relay nodes swap in a string topology", "chain on the fly in gridto-string topology", "circular and parallel movements in a grid" etc. ${ }^{[2]}$ Outdoor experiments consider node placement based on environmental suitability for wireless communication. On the other hand, random node placements and movements are often suitable for indoor experiments. Thus, two strategies are generally considered: "controlled" and "random node placement plus movement". Artificial traffic generated by traffic generators following specific distributions are sometimes used to demonstrate real-time applications over MANET, and maximum or best-case performances are evaluated. A detailed discussion on node placement, movement and traffic patterns for real-life MANET experimentations can be found in [2].

For real-life MANET experiment, a pure multi-hop ad hoc network consisting of four mobile nodes (i.e., laptops) has been considered and four specific topologies which are shown in Fig. 1 have been explored. Under the "string node placement" category, i.e., Scenario-1 to Scenario-3, static and roaming nodes as well as end-node swap scenarios have been considered. In the "grid and hybrid node placement" category, i.e., Scenario-4, a string-grid-string topology has been evaluated.

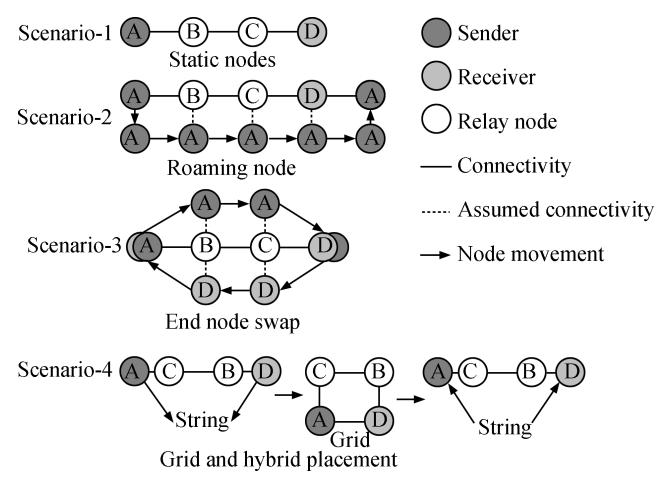

Fig. 1 Node topology and movement patterns for experimental and simulation cases

For these four specific scenarios, six individual cases have been investigated to evaluate the typical performance metrics like throughput and packet delivery ratios (PDR). Table 1 lists the cases, scenarios and protocols which are evaluated in the real experiments and corresponding simulations. For cases 1, 2, 3 and 4, ICMP Ping application has been used in the experiments, as it is quite simple and reflects the end-to-end network connectivity in a typical unreliable MANET. In the simulation, the constant bit rate (CBR) traffic over UDP with similar packet size is considered for cases 1 to 4 to achieve similar traffic properties. For cases 5 and 6 , a streaming video transmission which utilises HTTP over TCP connection is considered in real experiments, and file transfer protocol (FTP) over TCP is used in simulation as the application characteristics are much similar based on 
Table 1 Experimental and simulation cases for evaluation of MANET

\begin{tabular}{|c|c|c|c|c|c|c|}
\hline \multirow{2}{*}{ Case } & \multirow{2}{*}{ Scenario } & \multicolumn{2}{|c|}{ Network protocol } & \multirow{2}{*}{$\begin{array}{c}\text { Experiment/simulation } \\
\text { time (s) }\end{array}$} & \multirow[t]{2}{*}{ Traffic source } & \multirow{2}{*}{$\begin{array}{c}\text { Traffic } \\
\text { destination }\end{array}$} \\
\hline & & Experiment & Simulation & & & \\
\hline Case 1 & Scenario-1 & ICMP ping & CBR over UDP & 300 & Node A & Node D \\
\hline Case 2 & Scenario-2 & ICMP ping & CBR over UDP & 120 & Node A & Node D \\
\hline Case 3 & Scenario-3 & ICMP ping & CBR over UDP & 120 & Node A & Node D \\
\hline Case 4 & Scenario-4 & ICMP ping & CBR over UDP & 120 & Node A & Node D \\
\hline Case 5 & Scenario-1 & HTTP over TCP & FTP over TCP & 180 & Node A & Nodes B, C, D \\
\hline Case 6 & Scenario-1 & HTTP over TCP & FTP over TCP & 300 & Node A & Node D \\
\hline
\end{tabular}

connection establishment, maintenance and data transfer situations.

The ICMP ping application (as in Windows operating system) has been set out to generate 100 requests of 1500 bytes of data in bidirectional fashion from both node $\mathrm{A}$ and D, which exhibits UDP like performance evaluation over MANET for cases 1, 2, 3 and 4. For test case 1, it has been found in several repeated examinations that it took approximately $300 \mathrm{~s}$ for the source node (i.e., node A) to send out 100 ICMP requests to the destination node successfully as shown in Table 1. Similarly, for test cases 2 to 4 , it has been observed that approximately $120 \mathrm{~s}$ are required to generate 100 ICMP request packets from the source node to the destination node successfully.

For TCP performance evaluation, node A transmits streaming video, and the both relay nodes $\mathrm{B}$ and $\mathrm{C}$ are permitted to receive the video along with the destination node $\mathrm{D}$ for $180 \mathrm{~s}$ (arbitrarily taken) in case 5 . On the other hand, only node D can receive the streaming video for $300 \mathrm{~s}$ (arbitrarily taken) in case 6 , and nodes $\mathrm{B}$ and $\mathrm{C}$ are simply relay nodes. In all the six cases, network data packets have been captured using Wireshark protocol analyser ${ }^{[23]}$ for analysis.

\subsection{Setup and parameters of real-life ex- perimentation}

Usually, simulation works often consider theoretical models, e.g., radio propagation and mobility models which do not actually reflect real-world observations. One of the focuses of this paper is to identify the facts and issues to develop a more realistic simulation model for MANET.

For both the experiments and simulations, optimised link state routing (OLSR) is used as the routing protocol for the MANET. A real-life implementation of OLSR protocol Olsrd $^{[24]}$ has been used on four laptops to investigate the cases listed in Table 1. Olsrd supports Windows, UNIX, OSX, BSD, Android, etc. operating systems, and runs on almost any wireless local area network (WLAN) card that typically supports ad hoc networking. It occupies very little CPU power and is highly scalable. A screenshot of Olsrd and the default parameter settings that have been used in all the real experiments of this paper are shown in Fig. 2 (a). The experimental setup of a 2-hop 4 node wireless connectivity is depicted in Fig. 2 (b). A description of the network nodes used in the experiments and environmental conditions are given in Table 2.

It can be noted that laptops with different types of net- work adapters are considered as it is an obvious case for considering any wireless network in real world. Although different adapters from different vendors may raise heterogeneity, all the adapters in the experiments are configured to follow the default IEEE $802.11 \mathrm{~g}$ standard parameters, e.g., transmission power, receiver sensitivity. Therefore, it can be claimed that the heterogeneity of network adapters will not make significant deviation in the outcome of the performance evaluation.

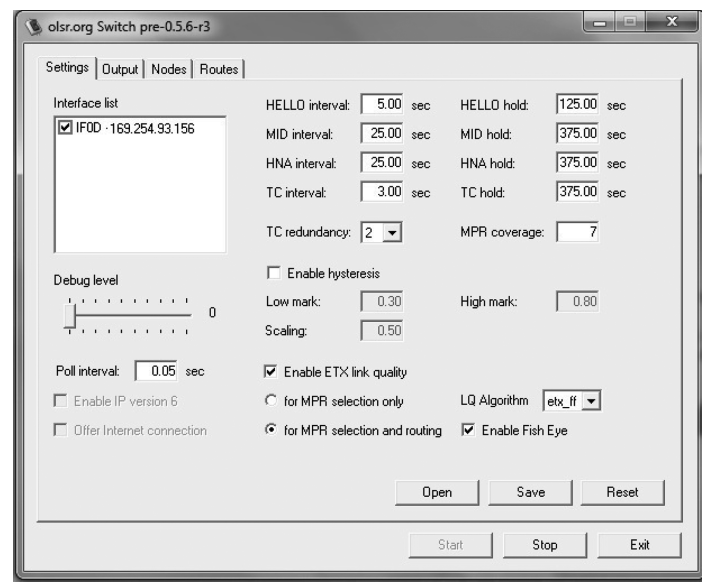

(a) A screenshots of the Olsrd tool

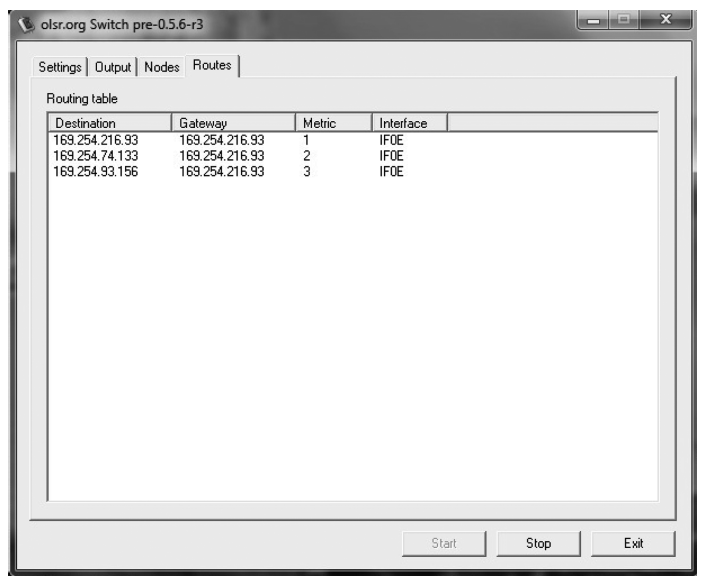

(b) A screenshot of Olsrd tool showing 2-hop node connectivity from node $\mathrm{A}$ to node $\mathrm{D}$

Fig. 2 Screenshots of the olsrd tool

For cases 5 and 6 , Broadcam ${ }^{[25]}$, a freely available broadcasting and video streaming server, has been used. It 
Table 2 Packet generation time for 100 ICMP packets in real experiment

\begin{tabular}{|c|c|}
\hline Parameters & Values \\
\hline Node type & Laptop with node mobility $0.5 \mathrm{~m} / \mathrm{s}$ \\
\hline \multirow[t]{2}{*}{ Operating system } & Windows XP 32bit (Node B and D) and Windows 764 bit (Nodes A and C) \\
\hline & $\begin{array}{l}\text { Node A: Realtek RTL8191SE wireless LAN 802.11n peripheral component } \\
\text { Interconnect express (PCI-E) }\end{array}$ \\
\hline \multirow[t]{3}{*}{ Network interface card (NIC) } & Node B: Broadcom $802.11 \mathrm{~g}$ wireless LAN \\
\hline & Node C: DELL wireless 1397 WLAN mini card \\
\hline & Node D: acer inviLink $802.11 \mathrm{~b} / \mathrm{g}$ \\
\hline Network type & Ad hoc with no authentication mechanism, IEEE $802.11 \mathrm{~g}$ compatible \\
\hline \multirow[t]{2}{*}{ Link speed } & 54-36 Mbps wireless links with no control mechanism \\
\hline & Node A: 169.254 .172 .66 \\
\hline \multirow{4}{*}{ IPv4 address } & Node B: 169.254 .216 .93 \\
\hline & Node C: 169.254 .74 .133 \\
\hline & Node D: 169.254 .93 .156 \\
\hline & Node $\mathrm{A} \rightarrow \mathrm{B}: 37$ meters \\
\hline \multirow[t]{3}{*}{ Distance (60\% successful packet reception) } & Node $\mathrm{B} \rightarrow \mathrm{C}: 43$ meters \\
\hline & Node $\mathrm{C} \rightarrow \mathrm{D}: 45$ meters \\
\hline & Total distance between node $\mathrm{A} \rightarrow \mathrm{D}: 125$ meters \\
\hline Environment & Open field with very few trees and no buildings, warm and sunny day \\
\hline
\end{tabular}

Table 3 Ns-2 PHY and MAC layer simulation parameters

\begin{tabular}{ccccc}
\hline \multirow{2}{*}{ Node ID } & Case & $\begin{array}{c}\text { First ICMP request } \\
\text { send time (s) }\end{array}$ & $\begin{array}{c}\text { Last ICMP request } \\
\text { send time (s) }\end{array}$ & $\begin{array}{c}\text { Average ICMP packet } \\
\text { generation time (s) }\end{array}$ \\
\hline \multirow{3}{*}{ Node A } & Case 1 & 6 & 287 & 2.81 \\
& Case 2 & 9 & 124 & 1.15 \\
& Case 3 & 12 & 125 & 1.13 \\
\hline \multirow{3}{*}{ Node D } & Case 4 & 14 & 129 & 2.15 \\
& Case 1 & 2 & 269 & 1.11 \\
& Case 2 & 10 & 121 & 1.06 \\
& Case 3 & 13 & 119 & 1.14 \\
\hline
\end{tabular}

supports a graphical user interface (GUI) browser-based interface to stream and view live video images via HTTP over TCP.

Another interesting finding from our real experiment indicates that a distance of $35 \mathrm{~m}$ to $45 \mathrm{~m}$ is the maximum reach ability for individual mobile nodes, and it works well with the Olsrd tool to maintain a four node wireless ad hoc network. And with this distance, a maximum of $60 \%$ transmitted packets can be successfully received at the destination. Hence, we have considered this real world finding in our simulation by calibrating ns- 2 shadowing model parameters to reflect a maximum $60 \%$ packet reception at a distance between $35 \mathrm{~m}$ to $45 \mathrm{~m}$. However, this rate and distance may change in another network topological consideration, and it will obviously require recalibration depending on the particular circumstance.

After analysing the captured packets by Wireshark, the packet generation times or intervals for case 1 to case 4 are computed shown in Table 3. Usually, ICMP packets are generated at a steady rate, e.g., in every second. However, in this real experiment it is found that it is fluctuating over the time due to the "Request time out" period to receive a corresponding reply from the destination node. To capture this phenomenon in the simulation we have introduced corresponding delay value based on Table 3 for CBR over UDP packet generation.

\subsection{Simulation setup and parameters}

The popular network simulator ns-2 with 80211Ext extension $^{[26]}$ is used to develop the 6 corresponding simulations which are listed in Table 1. All the latest bug fixes have been applied to get optimum output. The $\mathrm{UMOLSR}^{[27]}$ which is a popular version of OLSR protocol has been used in the simulation. Four network nodes of the same type and same network interface card (NIC) configuration have been used to simplify the overall simulation process along with all default IEEE $802.11 \mathrm{~g}$ parameters. Several Olsrd parameters, e.g., Willingness = 3, HELLO INTERVAL $=5 \mathrm{~s}$ and TC_INTERVAL $=3 \mathrm{~s}$ have been adjusted in UM-OLSR to produce the closest match with the configuration of Olsrd tool that has been used for the real- 
world experiments.

A Cisco Aironet $802.11 \mathrm{a} / \mathrm{b} / \mathrm{g}$ wireless network card has been chosen for this research. The receiver sensitivity of the card is set to $-71 \mathrm{dBm}$ and transmit power of $15 \mathrm{dBm}$ at $54 \mathrm{Mpbs}$ data rate using quadrature amplitude modulation 64 bit (QAM64) scheme over IEEE $802.11 \mathrm{~g}^{[28]}$. We choose this particular wireless network card as the vendor explicitly mentions its receiver sensitivity and transmit power for different data rates within its specification.

Ns-2 simulation is applied using shadowing propagation model based on the real experiment information given in Table 2 to generate case 1 given in Table 1 . The distance between two consecutive nodes is set to $40 \mathrm{~m}$ (as a generic consideration), and shadowing model parameters, i.e., path loss exponent, $\beta$, shadowing deviation, and $\sigma_{d B}$ are tuned to find the receiver threshold value to achieve $60 \%$ successful packet reception (as explained earlier) as given in Table 1. In the tuning process, three sets of values are investigated. In Set-1, $\beta$ is kept to 2.8 and $\sigma_{d B}$ is varied from 6.0 to $5.3 \mathrm{~dB}$. In Set- $2, \sigma_{d B}$ is kept at $6.0 \mathrm{~dB}$ and $\beta$ is varied from 2.8 to 2.1. Finally, in Set-3, both $\beta$ and $\sigma_{d B}$ are varied from 2.8 to 2.1 and 6 to $5.3 \mathrm{~dB}$. Each set is tested in ns-2 simulation environment and results are analysed to see whether $60 \%$ of data packets are correctly received at both node $\mathrm{A}$ and node D. Finally, $\beta=2.3$ and $\sigma_{d B}=6.0 \mathrm{~dB}$ produce a successful packet reception rate of $60 \%$ at a distance of $40 \mathrm{~m}$ with receiver threshold value of $4.55663 \mathrm{E}-10 \mathrm{~W}$ or $-63 \mathrm{dBm}$. Therefore, $\beta=2.3$ and $\sigma_{d B}=$ $6.0 \mathrm{~dB}$ have been used in the simulations to closely model the real world experiments. The overall calibration process is done using the propagation.cc file provided by ns- 2 in "-ns-2.34/indep-utils/propagation" directory.

Based on the packet generation time given in Table 3,
$100 \mathrm{CBR}$ requests of size 1500 bytes are generated in ns-2 simulation using UDP for case 1 to case 4 . However, in ns- 2 the default inter-burst transmission interval is set to $3.44 \mathrm{~ms}$ which is much smaller than the packet generation time given in Table 3. Hence, it produces much higher data throughput than the real experimentation results. Therefore, ns-2 transmission interval is adjusted to produce packet generation time given in Table 3 for cases 1 to 4 . For cases 5 and 6 , TCP window and packet size are set to 8192 and 1460 bytes, respectively in the simulation based on the Wireshark trace analysis of real experiments.

It is noted that for case 1 to case 4 , experimental results produce two fragments of sizes 1514 bytes and 62 bytes for a 1576 bytes frame. On the other hand, ns- 2 simulation shows fragments of 1020 bytes and 520 bytes for a 1540 bytes frame. Therefore, 1500 bytes of ICMP ping and 1500 bytes of CBR packets show 76 bytes and 40 bytes of overhead in experiments and simulation, respectively. This will usually tend to produce slightly better results for simulation than the experiments. For the experiment, only ICMP requests are considered (as discussed earlier in Section 3.2) in the throughput measurement to make similar comparative results with CBR over UDP transmission in simulation as CBR protocol does not have reply functionality from the receiver like ICMP.

In simulation, values of typical physical (PHY) and medium access control (MAC) layer parameters, e.g., header duration, CWMin, CWMax, slot time and SIFS values are set based on the IEEE $802.11 \mathrm{~g}$ specification. The value of preamble capture is set to true similar to the NIC configuration of experimental mobile nodes, i.e., laptops. The detailed parameter settings for the overall simulation are given in Table 4 .

Table 4 ns-2 PHY and MAC layer simulation parameters

\begin{tabular}{|c|c|}
\hline Parameters & Values \\
\hline Ns-2 version & 2.34 \\
\hline PHY and MAC layer & IEEE $802.11 \mathrm{~g}$ with 80211 PHYEXT \\
\hline Frequency & $2.4 \mathrm{GHz}$ \\
\hline Propagation model & Shadowing with path loss; $\beta=2.3, \sigma_{d B}=6.0$ for $40 \mathrm{~m}$ distance and $60 \%$ correct packet reception rate \\
\hline \multicolumn{2}{|c|}{ PHY layer parameters } \\
\hline Receiver sensitivity & $-71 \mathrm{dBm} @ 54 \mathrm{Mbps}$ for IEEE $802.11 \mathrm{~g}$ \\
\hline Transmit power & $15 \mathrm{dBm} @ 54 \mathrm{Mbps}$ for IEEE $802.11 \mathrm{~g}$ \\
\hline Receiver threshold & $-63 \mathrm{dBm}$, calculated based on the propagation model \\
\hline Modulation scheme & QAM64 \\
\hline Header duration & $20 \mu \mathrm{s}$ \\
\hline Preamble capture & True \\
\hline \multicolumn{2}{|c|}{ MAC layer parameters } \\
\hline Maximum data rate & $54 \mathrm{Mbps}$ with basic data rate $6 \mathrm{Mbps}$ \\
\hline CWMin & 15 \\
\hline CWMax & 1023 \\
\hline Slot time & $9 \mu \mathrm{s}$ \\
\hline Short inter-frame space (SIFS) & $10 \mu \mathrm{s}$ \\
\hline \multicolumn{2}{|l|}{ Other parameters } \\
\hline Antenna type & Omni-directional with antenna height $1.5 \mathrm{~m}$ \\
\hline UDP packet size & 1500 bytes, CBR over UDP \\
\hline TCP packet size & 1460 bytes with window size 8192, FTP over TCP \\
\hline Routing protocols & UM-OLSR \\
\hline
\end{tabular}




\subsection{Performance metrics and evaluation criteria}

A number of performance metrics can be considered to evaluate UDP and TCP over real-life MANET, e.g., throughput, packet delivery ratio (PDR), end-to-end (E2E) delay, roundtrip transmission time (RTT), TCP window size. For real-time applications, e.g., streaming audio and video, typical MAC and PHY, layer performance metrics, e.g., bit error rate (BER), signal-to-noise ratio (SNR), queue performance, packet scheduling, priority can be very helpful to create realistic simulation environment ${ }^{[2]}$. To judge the performance of the overall network, data throughput and PDR have been considered for both UDP and TCP transmission in this paper.

\section{Experimental results and analysis}

For simple comparisons, primarily data throughput is considered in all the 6 cases for both experiments and simulations in Sections 4.1 to 4.6. Statistical results for PDR are presented in Section 4.7.

\subsection{Case 1: String topology with static nodes}

In case 1 , all the 4 mobile nodes are set in a static string network topology which is a generic worst case scenario where mobile nodes are in their maximum reachability range. Frequent fluctuations are observed in both experiment and simulation of case 1 as shown in Fig. 3 (a) and Fig. 3 (b) due to unstable end-to-end wireless links between node A and D. From the results, it can be observed that the average throughput performances are in similar pattern for both experiment and simulation. Experimental results show low frequency of fluctuations, as ICMP requests are sent and the nodes wait for a time-out period to receive the replies. On the other hand, CBR packets are constantly sent based on the interval periods given in Table 3. Hence, the simulation shows higher frequency of packet generation.

\subsection{Case 2: String topology with roaming node}

In case 2 , both experimental and simulation results exhibit more stable throughputs than case 1 which are shown in Fig. 4 as node A starts moving towards node D gradually depicted in Fig. 1. According to Fig.4(a), experiment shows a drop in throughput between time $60 \mathrm{~s}$ to $80 \mathrm{~s}$ while node A passes over the relay nodes and frequent ICMP redirection occurs due to OLSR routing table updates. But this particular incident is not clearly visible in simulation as CBR packets are either received or dropped but not redirected as in the experiment. The initial fluctuations between $10 \mathrm{~s}$ to $20 \mathrm{~s}$, observed in both Fig. 4 (a) and Fig. 4 (b), are due to route establishments. The overall average throughputs show similar pattern. In this particular case, it is clear that mobility actually helps the network performance to overcome from uncertainty.

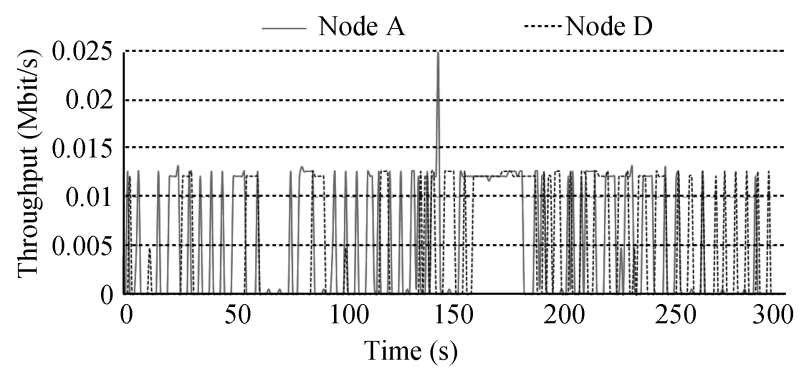

(a) Case 1: ICMP throughput performance in real experiment

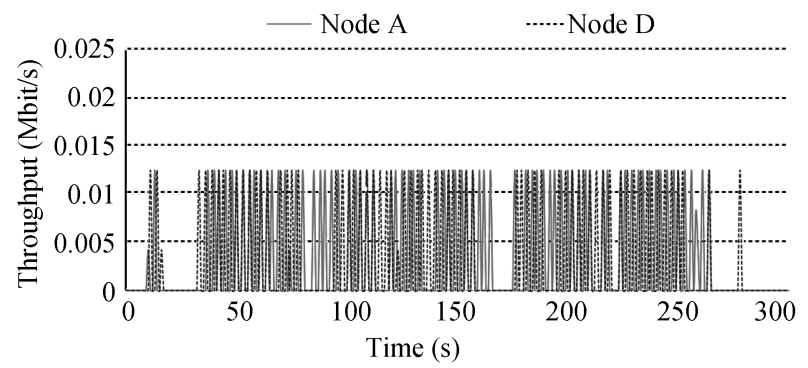

(b) Case 1: CBR over UDP throughput performance in simulation

Fig. 3 Comparison of experiment and simulation for case 3

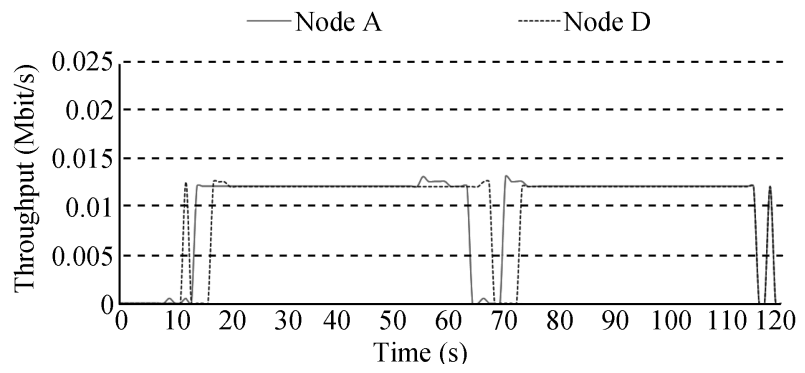

(a) Case 2: ICMP throughput performance in real experiment

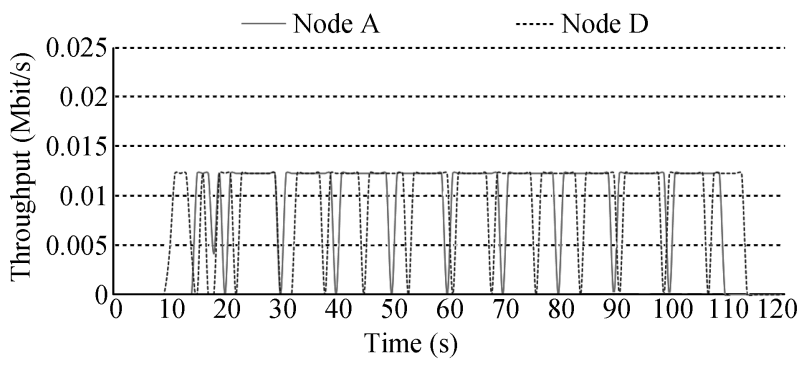

(b) Case 2: CBR over UDP throughput performance in simulation

Fig. 4 Comparison of experiment and simulation for case 2

\subsection{Case 3: String topology with end nodes swap}

In case 3 , both nodes $\mathrm{A}$ and $\mathrm{D}$ are moving towards each other from opposite directions as shown in Fig. 1. The experimental and simulation results, shown in Fig. 5 (a) and Fig. 5 (b), respectively exhibit more stable performance compared to case 2, i.e., Fig. 4 (a) and Fig. 4 (b). It is noted 
that mobility on both end nodes overcomes the sudden drop between time $60 \mathrm{~s}$ and $70 \mathrm{~s}$ in throughput which occurs for case 2 experiment. The effect of routing table update does not show any significant impact. However, a more detailed network layer performance analysis is necessary in this regard. Again, overall average throughputs show close similarity in both experiment and simulation. From our real network experiment, it can be observed that it is quite crucial to track routing table updates for OLSR as the both end nodes are moving towards each other at a constant rate and sometimes try to communicate directly by passing the relay nodes.

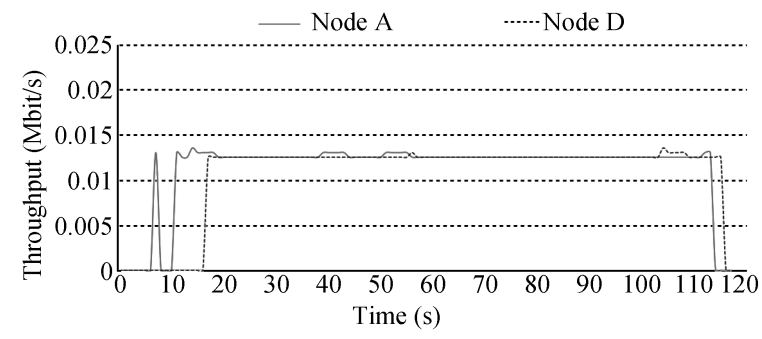

(a) Case 3: ICMP throughput performance in real experiment

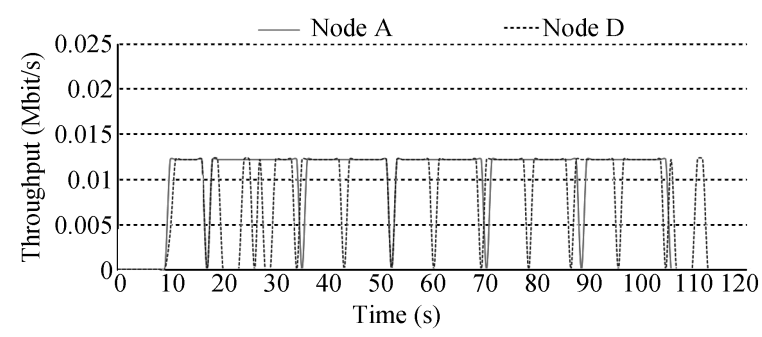

(b) Case 3: CBR over UDP throughput performance in simulation

Fig. 5 Comparison of experiment and simulation for case 3

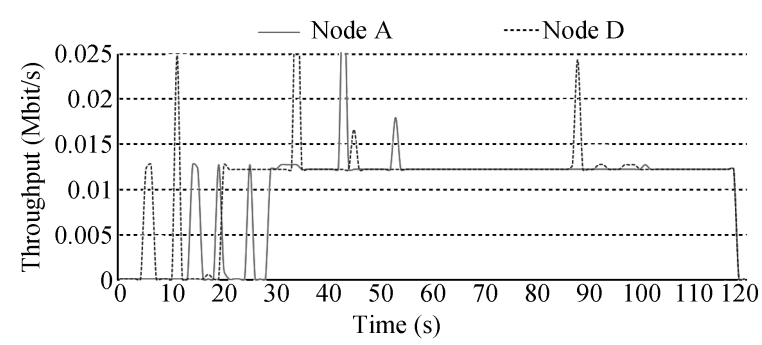

(a) Case 4: ICMP throughput performance in real experiment

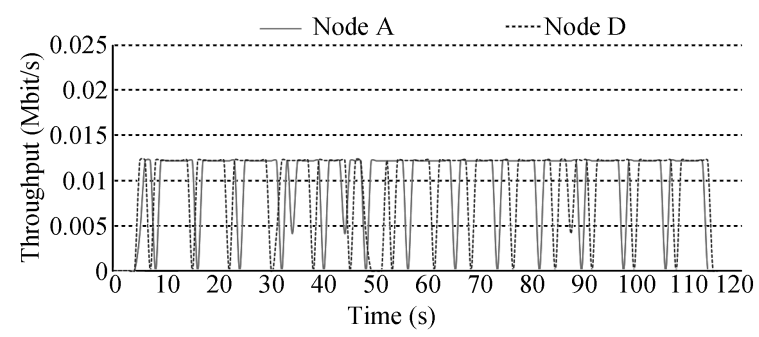

(b) Case 4: CBR over UDP throughput performance in simulation

Fig. 6 Comparison of experiment and simulation for case 4

\subsection{Case 4: Hybrid topology with String- Grid-String formation}

In case 4 , both nodes $\mathrm{A}$ and $\mathrm{D}$ move in such a way that creates a string-grid-string topology as shown in Fig. 1. Experimental results shown in Fig. 6 (a), shows several spikes in the throughput results at time of string-grid ( $30 \mathrm{~s}$ to $50 \mathrm{~s})$ and grid-string ( $80 \mathrm{~s}$ to $100 \mathrm{~s}$ ) formation due to frequent ICMP redirection and route table update. However, according to Fig. 6 (b), unusual throughput fluctuations are observed in the mentioned time periods for simulation. It can be noted that, during the grid form period, nodes A and $\mathrm{D}$ are in direct communication range of each other. Therefore, more stable throughput results are seen from the experimental results. Again, as in the previous cases, average throughput performances show similarity in pattern for both experimentation and simulation. It is understood that ICMP redirection, IP packet fragmentation, etc. in the real world scenario can create an obvious difference compared to the simulation results. Thus, it is worth mentioning that researchers should pay particular focus to the protocol level implementation in synthetic network traffic generation (like by using iperf, BWPing, httping, etc.) and use simulations consistent/equivalent to real experimentations.

\subsection{Case 5: String topology with unre- stricted relay nodes}

In case 5, TCP based video data over HTTP is received by node $\mathrm{D}$ as well as captured and forwarded by relay nodes $\mathrm{B}$ and $\mathrm{C}$. Therefore, in this experiment, sender node A rarely receives actual data request from the end node $\mathrm{D}$ due to poor network connectivity in this worst case scenario, though $\mathrm{D}$ receives moderate retransmitted TCP packets from the relay nodes up to $100 \mathrm{~s}$ as shown in Fig. 7 (a). At almost $120 \mathrm{~s}$, A starts to receive TCP request packets from $\mathrm{D}$ and responds thereafter. According to Fig. 7 (b), sender node A initially is able to capture node $\mathrm{D}^{\prime}$ s requests but similarly due to the network congestion created by the relay nodes, node A receives very few TCP packets from $\mathrm{D}$ in simulation. In both the experiment and simulation, average throughput is below the minimum expected level for requesting and receiving streaming data over MANET. From our experiment in case 5 , it has been observed that it is quite difficult for the destination node $\mathrm{D}$ to establish and maintain an end-to-end TCP connection with the source node $\mathrm{A}$. This is because the relay nodes $\mathrm{B}$ and $\mathrm{C}$ are also sending request and responses back to the source node.

\subsection{Case 6: String topology with re- stricted relay nodes}

In case 6 , both experimental and simulation time have been increased to $300 \mathrm{~s}$ to allow proper end-to-end TCP packet transmission and acknowledgement reception. Node $\mathrm{D}$ is the only destination and the relay nodes $\mathrm{B}$ and $\mathrm{C}$ are not allowed to request streaming data from sender A. Experimental results show that both nodes A and D start receiving TCP packets from each other from the very beginning and after $120 \mathrm{~s}$ the results show better throughput performance which is depicted in Fig. 8 (a) compared to case 5 . In simulation, after a late start, both $\mathrm{A}$ and $\mathrm{D}$ start ex- 
changing packet data in a fluctuating fashion, although after $200 \mathrm{~s}$ it shows moderate throughput performance. The average throughput performance is much higher than the experimental result in case 5 and 6 which needs more investigation.

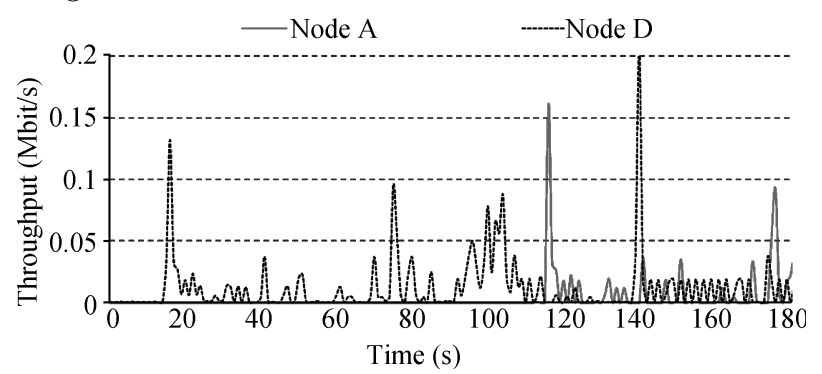

(a) Case 5: TCP throughput performance in real experiment

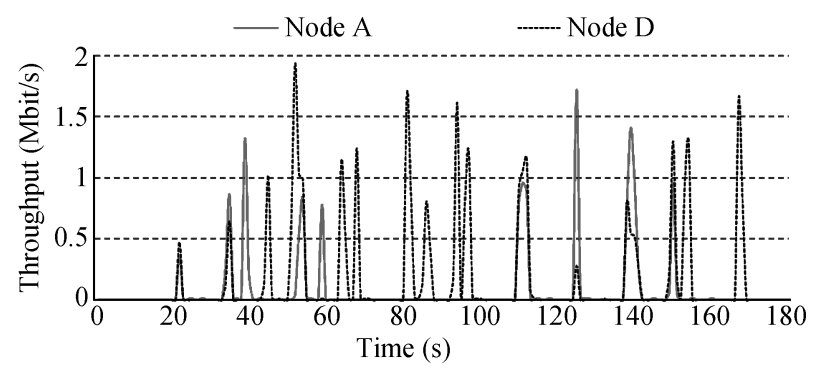

(b) Case 5: TCP throughput performance in simulation

Fig. 7 Comparison of experiment and simulation for case 5

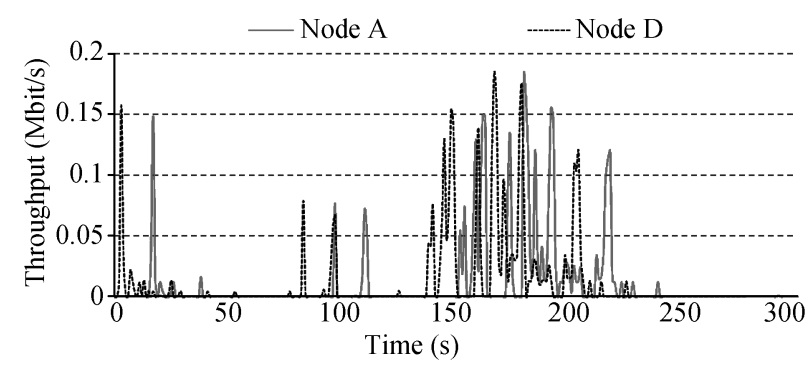

(a) Case 6: TCP throughput performance in real experiment

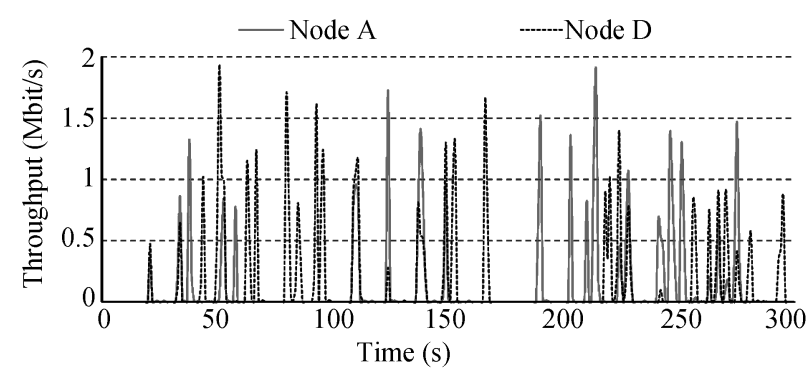

(b) Case 6: TCP throughput performance in simulation

Fig. 8 Comparison of experiment and simulation for case 6

\subsection{Statistical results from real- experiment and simulation}

Table 5 shows the average percentage of PDR for all six experiments and corresponding simulations. Although the parameters of the simulations are set very carefully to reflect the real world experiments, the corresponding simula- tions exhibit much lower PDR for case 1 to 4 . On the other hand, the simulation PDR shows higher value than the real experiments for cases 5 and 6 . To investigate this anomaly, the average throughput and average packet generation rate for cases 5 and 6 are analysed, and the obtained values are given in Table 6.

The packet generation rate of the simulations is adjusted in Section 3.3 based on the Wireshark trace information given in Table 3 for cases 1 to 4 . Unfortunately, for cases 5 and 6 , the appropriate value for packet generation cannot be set for the FTP over TCP in corresponding simulations based on the Wireshark trace analysis. By investigating the statistical results, it is found that the average packet generation rate is much higher in simulation than that of the experiments. Hence, the average PDR and network throughput in simulation show much better results than the experiments for cases 5 and 6 .

Table 5 Average percentage of RDR for experiments and corresponding simulations

\begin{tabular}{ccc}
\hline Case ID & $\begin{array}{c}\text { Average percentage of } \\
\text { PDR (Experiment) }\end{array}$ & $\begin{array}{c}\text { Average percentage of } \\
\text { PDR (Simulation) }\end{array}$ \\
\hline Case 1 & 60.0 & 33.1787 \\
Case 2 & 96.5 & 62.5912 \\
Case 3 & 96.0 & 64.5833 \\
Case 4 & 95.0 & 65.9498 \\
Case 5 & 80.5 & 89.7047 \\
Case 6 & 85.3 & 89.327 \\
\hline
\end{tabular}

\section{Lessons learnt from reality}

Based on the analysis and findings presented in this paper, several important aspects have been identified which can be useful for further real-life experiments and preparing guidelines for simulation based evaluations.

In the simulations presented in this paper, all of the network nodes have been chosen to be the same which is not practical, as in the real world, it is difficult to find identical mobile nodes. Different node profiles and NIC configurations can be created to overcome this limitation in the simulations. It was also noted that Windows operating system based machines usually prefer to use the same private block of IP address every time it joins an ad hoc network.

Although shadowing propagation model performs better than other conventional models such as Free Space and Two Ray Ground ${ }^{[29]}$, Nakagami-M distribution model ${ }^{[30]}$ has offered more configurable parameters to correctly model the realistic scenarios. Many network simulators, e.g., ns-2 $2^{[11]}$, NCTUns ${ }^{[31]}$, QualNet ${ }^{[32]}$. also offer real-life experimental parameter settings for specific scenarios, e.g., urban, shadowed urban, indoor, office, public place of interest. which are also helpful in this regard.

Configuring PHY layer parameters, such as receiver sensitivity, i.e., career sense threshold, individual transmission power, wireless channel and modulation technique selection are very difficult to set according to the popular wireless standards and specifications. In the real world, different manufacturers use different settings for their products and they rarely disclose low level parameters which might be required to model a realistic simulation. Furthermore, MAC layer values, e.g., packet header duration, frame format, in- 
Table 6 Average throughput and packet generation rate for experiments and corresponding simulations for cases 5 and 6

\begin{tabular}{cccc}
\hline Case ID & $\begin{array}{c}\text { Average network throughput } \\
(\mathrm{Mbps}) \text { (Experiment) }\end{array}$ & $\begin{array}{c}\text { Average network throughput } \\
(\mathrm{Mbps}) \text { (Simulation) }\end{array}$ & $\begin{array}{c}\text { Average generated packet (s) } \\
\text { (Experiment) }\end{array}$ \\
\hline Case 5 & 0.000382492 & 0.122376 & 4.757 \\
Case 6 & 0.000855595 & 0.115220 & 4.139 \\
\hline
\end{tabular}

dividual field structures, modulation schemes, bandwidth settings, etc. widely vary according to the specific wireless PHY and MAC standards (e.g., IEEE802.11a/b/g/e/n/pdraft, IEEE802.11-2007).

In link layer management, queue length and its properties are important issues which greatly influence multi-hop ad hoc network performance. Real-time applications, e.g., video streaming can evaluate TCP performance and highly depend on efficient packet queue management and related algorithms.

It has been observed from the practical experiments that a large amount of time is required to join an offered ad hoc network and establish connectivity. Furthermore, network split and merge operations are very slow, this creates bottlenecks for real time applications. Although, the main track for future reactive and proactive MANET routing protocols has been already identified for general-purpose MANET, cross-layer interaction of a routing agent is still lacking. From the practical examination, it can be observed that due to the lack in notification of topological change from lower layer of network protocol stack, the time required to update routing table and readjust parameters is often high which creates performance degradation.

Traditional transport layer protocols for real-time applications, e.g., TCP, are mostly developed for wired and infrastructural wireless networks and are not always suitable for MANET ${ }^{[33]}$. From the experiments, it is seen that due to the lack of interaction between transport and network layer protocols, TCP synchronisation, retransmission and redirections occurred heavily. As transport layer protocols are not aware of the lower level topological changes, they assume network congestion and employ corresponding actions most of the time. Although many simulation-based researches with TCP evaluation have been executed, very few have considered realistic environmental settings and only few have been done in a real-world scenario ${ }^{[18,33]}$. TCP performance evaluation over a real MANET has significant value in the research and development of real time applications, control system and automation based on multi-hop ad hoc networking.

The wireless propagation modelling, physical layer parameter settings, MAC layer enhancement for better notification of link level change, adjustment of QoS, increasing capability of the routing protocols with cross layer interaction and transport layer protocol understanding and improvement are necessary to provide a generic baseline to develop MANET applications and services.

\section{Conclusion and future work}

A comprehensive investigation and analysis has been carried out in this paper with UDP and TCP over MANET using both real world experiments and simulation-based studies. Real-life OLSR protocol implementation has been used on Windows based machine with different types of NIC to reflect a typical and realistic MANET environment. To match with real world experiments OLSR implementation for ns-2 simulator has been used along with IEEE 802.11g PHY and MAC layer parameter settings with real world NIC parameter values and realistic propagation model tuning. In the analysis phase, similarities and other characteristics have been clearly identified.

It is understood that the regeneration of real time scenarios in simulation is very hard as it is often difficult to set all the necessary parameters accordingly. It is also found that node mobility can introduce better network performance for a multi-hop ad hoc network. This phenomenon is perceived by approximately $30 \%$ PDR improvements given in Table 5 when moving from case 1 (static network) to cases 2-4 (dynamic networks) for both experiment and simulation. It might be the case that the mobility allows routing protocols to find more efficient route than static scenarios. Therefore, to understand the true impact of mobility effect, it is necessary to introduce high traffic density along worst and generic test cases.

However, from the results of cases 5 and 6 it is strongly suggested that connection oriented protocols need more attentions and considerations in terms of parameter calibration which is in our consideration for future work.

Different wireless NIC implementation also signifies the effect of selecting appropriate receiver sensitivity and transmission power value. Although it is the vendor's responsibility to maintain the specifications as per the standardisation bodies, e.g., IEEE, it is also known that many vendors have implemented their product in a non-standard way and those types of equipments may create unwanted anomalies in scientific experiments.

Further case studies along with low level performance metric analysis and different topological models with mobility patterns will be considered in our future work. In these experiments, only throughput, and packet delivery ratios have been considered. Other performance analysis tasks, e.g., throughput measurement based on TCP window size, TCP congestion control mechanism, TCP retransmission process, etc. have been left for future work.

\section{References}

[1] M. Conti, S. Giordano. Multihop ad hoc networking: The theory. IEEE Communications Magazine, vol.45, no. 4, pp. 78-86, 2007.

[2] M. Conti, J. Crowcroft, A. Passarella. Multi-hop Ad Hoc Networks from Theory to Reality, New York, USA: Nova Science Publishers, Inc., 2007.

[3] J. M. M. Kamal, M. S. Hasan, A. L. Carrington, H. N. Yu. Lessons learned from real MANET experiments and simulation-based evaluation of UDP and TCP. In Proceedings of the 13th International Conference on Computer 
and Information Technology, IEEE, Dhaka, Bangladesh, pp. 511-515, 2010.

[4] M. Conti, S. Giordano. Multihop Ad Hoc networking: The reality. IEEE Communications Magazine, vol. 45, no. 4, pp. 88-95, 2007.

[5] D. Johnson, Y. Hu, D. Maltz. The Dynamic Source Routing Protocol (DSR) for Mobile Ad Hoc Networks for IPv4, [Online], Available: http://www.ietf.org/rfc/rfc4728.txt, May 05, 2011.

[6] D. A. Maltz, J. Broch, D. B. Johnson. Experiences designing and building a multi-hop wireless ad hoc network testbed, [Online], Available: http://citeseerx.ist.psu.edu/view$\mathrm{doc} /$ download?doi=10.1.1.70.8610\&rep $=$ rep1\&type $=$ pdf, Jun 9,2011

[7] K. W. Chin, J. Judge, A. Williams, R. Kermode. Implementation experience with MANET routing protocols. ACM SIGCOMM Computer Communication Review, vol. 32, no. 5, pp. 49-59, 2002.

[8] K. Konolige, C. Ortiz, R. Vincent, A. Agno, M. Eriksen, B. Limketkai, M. Lewis, L. Briesemeister, E. Ruspini, D. Fox, J. Ko, B. Stewart, L. Guibas. DARPA software for distributed robotics, [Online], Available: http: // www. ai.sri.com/centibots/papers/tech-report-dec2002.pdf, Jun17, 2011.

[9] B. Karp, H. T. Kung. GPSR: Greedy perimeter stateless routing for wireless networks. In Proceedings of the 6th Annual International Conference on Mobile Computing and Networking, ACM, New York, USA, pp. 243-254, 2000.

[10] R. S. Gray, D. Kotz, C. Newport, N. Dubrovsky, A. Fiske, J. Liu, C. Masone, S. McGrath, Y. G. Yuan. Outdoor experimental comparison of four ad hoc routing algorithms. In Proceedings of the 7th ACM International Symposium on Modelling, Analysis and Simulation of Wireless and Mobile Systems (MSWiM), ACM, New York, USA, pp.220-229, 2004.

[11] The Network Simulator - ns-2, [Online], Available: http:// www.isi.edu/nsnam/ns/, May 20, 2010.

[12] A. K. Saha, K. A. To, S. Palchaudhuri, S. Du, D. B. Johnson. Physical implementation and evaluation of ad hoc network routing protocols using unmodified simulation models. In Proceedings of SIGCOMM ASIA WORKSHOP, ACM, Beijing, China, 2005.

[13] S. Jadhav, T. Brown, S. Doshi, D. Henkel, R. Thekkeumnel. Lessons learned constructing a wireless ad hoc network test bed. In Proceedings of the 1st Workshop on Wireless Network Management, Trentino, Italy, 2005.

[14] C. A. Santivanez, R. Rananathan. Hazy Sighted Link State (HSLS) Routing: A Scalable Link State Algorithm, [Online], Available: http://www.ir.bbn.com/documents/techmemos/TM1301.pdf, August 7, 2010.

[15] R. Ramanathan, J. Redi, C. Santivanez, D. Wiggins, S. Polit. Ad hoc networking with directional antennas: A complete system solution. IEEE Journal on Selected Areas in Communications, vol. 23, no. 3, pp. 496-506, 2005 .

[16] T. Clausen, P. Jacquet. Optimized Link State Routing Protocol (OLSR), [Online], Available: http: //www.ietf.org/ rfc/rfc3626.txt, April 27, 2010
[17] C. E. Perkins, E. Belding-Royer, S. Das. Ad hoc OnDemand Distance Vector (AODV) Routing, [Online], Available: http://www.ietf.org/rfc/rfc3561.txt, May 6, 2011.

[18] E. Borgia, M. Conti, F. Delmastro, E. Gregori. Experimental comparison of routing and middleware solutions for mobile ad hoc networks: Legacy vs. cross-layer approach. In Proceedings of the 2005 ACM SIGCOMM Workshop on Experimental Approaches to Wireless Network Design and Analysis (E-WIND), ACM, New York, USA, pp. 8287, 2005.

[19] H. Lundgren, D. Lundberg, J. Nielsen, E. Nordström, C. Tschudin. A large-scale testbed for reproducible ad hoc protocol evaluations. In Proceedings of the Wireless Communications and Networking Conference, IEEE, Orlando, FL, vol. 1, pp. 412-418, 2002.

[20] P. Kumar, A. Krishnan. Throughput analysis of the IEEE 802.11 distributed coordination function considering erroneous channel and capture effects. In International Journal of Automation and Computing, vol. 8, no. 2, pp. 236-243, 2011.

[21] N. E. Wu, S. Thavamani, X. H. Li. Reliability and feedback of multiple hop wireless networks. International Journal of Automation and Computing, vol.4, no. 2, pp. 125$134,2007$.

[22] C. C. Hua, Y. Zheng, X. P. Guan. Modeling and control for wireless networked control system. International Journal of Automation and Computing, vol.8, no. 3, pp.357$363,2011$.

[23] Wireshark Foundation. Wireshark - Go Deep, [online] Available: http://www.wireshark.org/, May 15, 2010.

[24] A. Tønnesen, T. Lopatic, H. Gredler, B. Petrovitsch, A Kaplan, S. O. Tücke. Olsrd - An adhoc wireless mesh routing daemon, [Online], Available: http://www.olsr.org/, May 20, 2010

[25] NCH Software. Streaming Video Server Software Stream Live Video for the Web, [Online], Available: http://www.nchsoftware.com/broadcam/index.html, July 24,2010 .

[26] Q. Chen, F. Schmidt-Eisenlohr, D. Jiang, M. TorrentMoreno, L. Delgrossi, H. Hartenstein. Overhaul of IEEE 802.11 modeling and simulation in ns-2. In Proceedings of the 10th ACM Symposium on Modeling, Analysis, and Simulation of Wireless and Mobile Systems, ACM, New York, USA, pp. 159-168, 2007.

[27] MASIMUM. UM-OLSR, [Online], Available: http://masimum.dif.um.es/?Software:UM-OLSR, July 25, 2010.

[28] Cisco. Cisco Aironet 802.11a/b/g Wireless PCI Adapter, [Online], Available: http://www.cisco.com/en/US/prod/ collateral/wireless/ps6442/ps4555/ps5819/product_data sheet09186a00801ebc33.html, July 25, 2010.

[29] J. B. Andersen, T. S. Rappaport, S. Yoshida. Propagation measurements and models for wireless communications channels. IEEE Communications Magazine, vol.33, no.1, pp. 42-49, 1995

[30] M. Nakagami. The $m$-distribution, a general formula of intensity of rapid fading. Statistical Methods in Radio Wave Propagation, W. C. Hoffman, Ed., Oxford UK: Pergamon Press, pp. 3-36, 1960 
[31] S. Y. Wang, C. L. Chou. NCTUns tool for wireless vehicular communication network researches. Simulation Modelling Practice and Theory, vol. 17, no. 7, pp. 1211-1226, 2009.

[32] Scalable Network Technologies. QualNet, [Online], Available: http://www.scalable-networks.com/products/qualnet/, May 16, 2010.

[33] M. van der Schaar, P. A. Chou. Multimedia over IP and Wireless Networks: Compression, Networking, and Systems, USA: Elsevier Inc., 2007.

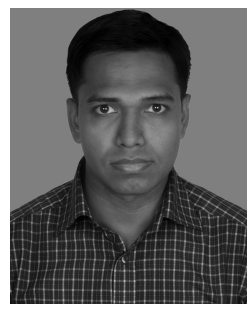

Joarder Mohammad Mustafa Kamal received B. Sc. degree in computer science and engineering from Military Institute of Science and Technology (MIST), University of Dhaka, Bangladesh in 2008, and M. Sc. degree in computing science from Staffordshire University, UK in 2011. Since 2012, he has been a $\mathrm{Ph}$. D. candidate in information technology at Monash University, Australia.

His research interests include wireless communication, mobile and cloud computing.

E-mail: Joarder.Kamal@monash.edu

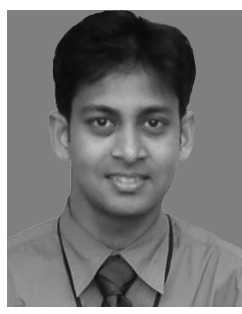

Mohammad Shahidul Hasan received his B.Sc. and M.Sc. degrees in computer science. He obtained his second M. Sc. degree in computer and network engineering from Sheffield Hallam University, UK and his Ph. D. degree at Staffordshire University, UK in networked control systems over mobile ad-hoc network (MANET). Currently, he is a full time lecturer and a member of the Mobile Fusion (MF) Applied Research Centre (ARC) at Staffordshire University.

His research interests include computer networks, networked control systems, remotely controllable mobile robot systems, real time systems, and wireless sensor networks.

E-mail: m.s.hasan@staffs.ac.uk (Corresponding author)

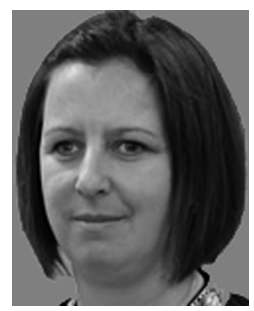

Alison L. Griffiths received both her M. Eng. and first B. Eng. (Hons) degrees from Staffordshire University, UK in 1999 and 1998, respectively. From 1999 to 2003 , she was a research associate on an Engineering and Physical Sciences Research Council (EPSRC) funded project whilst being a $\mathrm{Ph}$. D. candidate on the convergence of mobile computing and telecommunications at Staffordshire University. Since 2003, she has been a senior lecturer in telecommunications at Staffordshire University.

Her research interest includes mobile agents, cellular and IP packet switched networks, communication of different types of media (voice, video conferencing etc.), and control of mobile wireless ad-hoc networks.

E-mail: a.l.griffiths@staffs.ac.uk

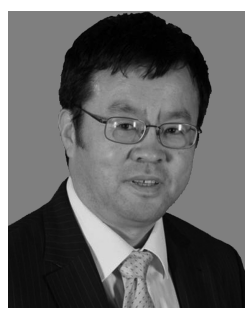

Hongnian $\mathbf{Y u}$ is currently a professor in computer science at Bournemouth University. He has extensive research experience in modelling, control of robots, mechatronics devices, neural networks, mobile computing, modelling, scheduling, planning and simulations of large discrete event dynamic systems, rodio frequency identification (RFID) with applications to manufacturing systems, supply chains, transportation networks, and computer networks. He has published over 200 research papers and held several grants from Engineering and Physical Sciences Research Council (EPSRC), the Royal Society, and other funding bodies. He is a member of the EPSRC peer review College and serves on various conferences and academic societies.

His research interests include mobile computing, modelling, scheduling, planning, and simulations of large discrete event dynamic systems with applications to manufacturing systems, supply chains, transportation networks, computer networks and RFID applications, modelling and control of robots and mechatronics, and neural networks.

E-mail: yuh@bournemouth.ac.uk 